\title{
ANALYTICAL INTELLIGENCE TOOLS FOR MULTICRITERIAL DIAGNOSTICS OF CNC MACHINES
}

\author{
Ivan Kuric ${ }^{1}$, Ivan Zajačko' ${ }^{1}$, Miroslav Císar ${ }^{1}$ \\ 1 Faculty of Mechanical Engineering, University of Žilina, Univerzitna 1, 010 26, Žilina, Slovakia, e-mail: ivan. \\ kuric@fstroj.uniza.sk, ivan.zajačko@fstroj.uniza.sk,miroslav.cisar@fstroj.uniza.sk
}

Received: 2016.08.31

Accepted: 2016.10.08

Published: 2016.12.01

\begin{abstract}
Analytical Intelligence is a set of methods and tools for acquisition and transformation of raw data into meaningful and useful information. Multicriterial diagnostics is an approach to obtain a real status of machining process just in time and produce a big pile of raw data. The paper presents a scheme of utilisation of analytical intelligence tools in multicriterial diagnostic of CNC machine tools. It is an effort to obtain a complex perception about all influences represented with measured data on machine precision.
\end{abstract}

Keywords: multicriterions diagnostics, analytical intelligence, data mining.

\section{INTRODUCTION}

Currently, we are seeing a significant rise in the modernization of processes and procedures across the whole spectrum of manufacturing companies in the engineering industry. This is a natural consequence of efforts to increase the efficiency of production processes primarily due to a reduction in production cost, reduction in energy consumption with emphasis on reducing environmental impacts caused by production itself, but also other dependent aspects, and also the efforts of manufacturing companies to ensure optimization of indirect costs associated with human resources, which are an integral part of costs. Another very important reason for modernizing production processes are continually increasing demands of customers for increasing the quality of production with demand for delivering in the required time and the required quantity. Engineering production companies are, due to diversification of customers, largely focused on the automotive industry and thus is also the associated requirement of their customer base to conform to standards as defined by ISO/TS 16949, which entails very high expectations for the quality of production. Because of this, multi-criteria diagnostics is very beneficial, based on analysis of the results obtained from manufacturers exact information about weaknesses in the processes of production and proposed solutions to existing or predictable problems can be made. An indirect consequence of globalization trends, is evident from all sectors of industry and economy, it is manifested by combining small and medium manufacturing enterprises to large structures that are decentralized in geographical nature, but have common leadership and share the same level of process control. This creates multinational corporations influencing several countries and their production influences the economy at international level. In such enterprises original manufacturers are transformed from autonomous production structures with complex production to highly specialised manufacturing units $[1,2,3]$.

The reason for the creation of specialized units is to ensure the maximum possible level of production quality and optimization of logistic processes within the group. However, this causes a new complication in the production process, the formation of hierarchical dependence of the manufacturing process where successive stages of production within the company are dependent on the production of the previous stages in the life cycle 
of production. For this reason, the occurrence of random and sudden interruptions of production or unplanned production decline is undesirable phenomenon with potentially serious repercussions for the entire production group. Prediction of the problems determined by the analysis of information obtained in production by obtaining measurable data is a very beneficial method of eliminating the aforementioned problems of manufacturing. Production processes are complex in nature with a large number of interacting actors. The results of mono-criteria diagnostics are useful only in analyzing an isolated property of the production process, or in the case in mono-criteria diagnostics of more complex property (e.g. vibrations) the result have broader scope but are susceptible to errors, due to misinterpretation of the results analyzed or the emergence of errors in cases where the process is affected by new actor that did not appeared previously and then "hidden" influence the result $[1,2,4,5]$.

Our aim is to obtain as realistic and comprehensive view of the manufacturing process as possible and to obtain measurement results that their analysis produce results with high diagnostic value with accurate description of the state of the manufacturing process. The data obtained through multi-criteria diagnosis have a very large content value. Depending on the way in which we look at the data, in a way of their further processing and, depending on the interpretation of the results obtained by the analysis of the collected data we can not only predict failures and the need of scheduled maintenance, but also predict other phenomenon related to the production and manufacturing process. With correct data analysis and correct interpretation of data we can create very precise results for optimization of energy requirements and environmental impacts of manufacturing processes, also we can optimize usage of human resources, and material flow, and other important factors. Because of wide application opportunities of the proposed multi-criteria diagnostics, and analysis of the results obtained we can apply the research results in a wide variety of manufacturing companies at the international level $[3,6,7]$.

Aim of our research is to ensure the development of analytical tools that process the data obtained through multi-criteria diagnostics to obtain the required answers to specific questions defined by mechanical engineering companies in the topic of increasing efficiency and optimiz- ing production processes. The resulting data set has a very large scope and the maximization of the content value, is however, dependent on a thorough knowledge of its structure and in particular in accordance with the dependencies observed in the gathered data. Production process is a continuous sequence of tasks and the various actions in the production process are not isolated factors affecting the final product, but are an integral set of mutually interacting factors that have both a direct impact on final product manufacturing, and also often lead to mutual interactions between each parameter by means of a visible but also hidden dependencies that need to be understood before of the processing and interpretation of the data obtained thru multicriteria diagnostics $[8,9,10]$.

\section{MULTICRITERIAL DIAGNOSTIC}

Frequently, only separate parameters of $\mathrm{CNC}$ machine tools in the framework of technical diagnostics are evaluated of. For example measurement of geometrical properties of worktable of CNC milling give good perception about precision about the worktable, however, it is an isolated and separated measurement (Fig. 1). Another example is the measurement of vibration of milling spindle (Fig. 1). These two separated measurement and two distinct evaluations give no relevant perception about a complex valuation precision of $\mathrm{CNC}$ machine tools. There are a lot of influences and parameters of machine tool which influence various precision parameters. Each of input parameters (e.g. temperature of spindle, vibration of spindle, vibration of worktable, geometrical property of worktable, etc.) influences on the output parameter (precision) by other way and other intensity. There is a complex effect inputs parameters for valuation of precision machine tools (Fig. 2). If we measure a lot of various data, the problem consists in relevant analysis and determination whose input parameters are significant for the evaluation of machine precision. Especially vibrations (V), temperature (T) and geometrical properties of individual machine units are measured and all these inputs influence various parameter of precision (P) differently. The application of analytical intelligence gives a possibility to obtain the size of influence among the measured and precision parameters (Fig. 2) [11, 12]. 


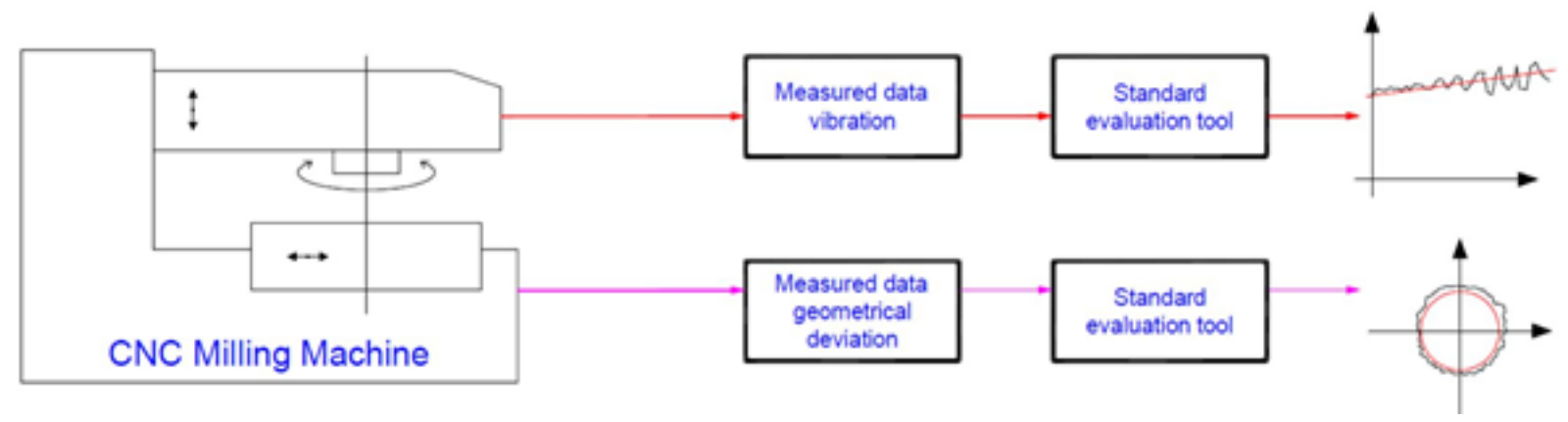

Fig. 1. Example of isolated measurent of parameters of CNC machine tools

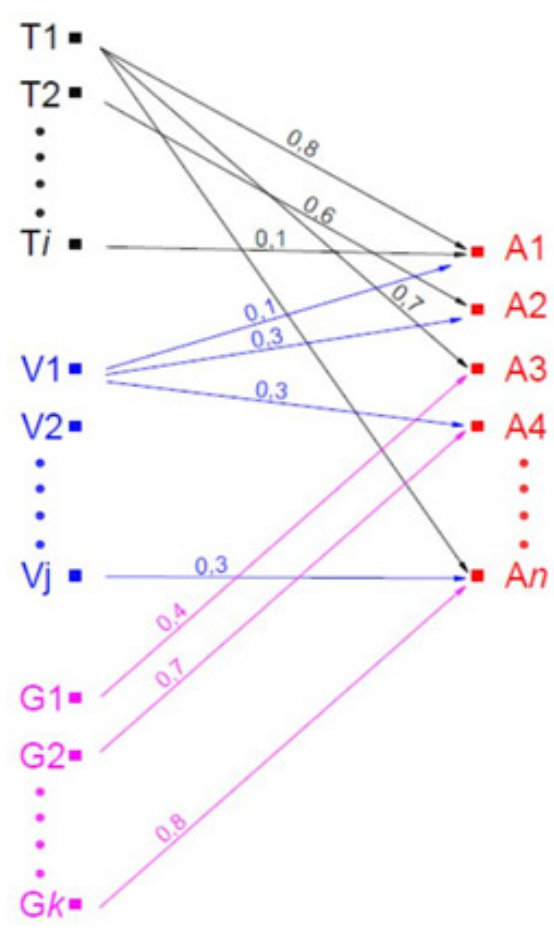

Fig. 2. Example of influence of measured input paraters of precision

With multi-criteria diagnostics of the production process we collect an extensive set of data in a relatively short time. We need very powerful analytical methods and tools to obtain applicable results in real time. Therefore, we focus our research on the implementation of advanced analytical methods for processing a large data set on comodity computing devices. We plan to achieve the desired performance when storing and subsequently processing the data collected from the production process by implementing the tools on top of database systems and subsequently ensure progressive implementation of data processing methods presently known under the term Data Mining. These methods and approaches are significantly contained in the tools some times known under the name of Analytical Intelligence. The applications of the general principles under which they carry out their tasks are already known. We will be able to deploy and modify them in order to achieve our goals with high efficiency.

The essence of muticriterial diagnostics is simultaneous usage of multiple methods of diagnostics in order to gain effects such as simplification of measurement, reducing time and costs necessary to perform measurements or to increase accuracy of measured data. Therefore, one of our aims is selection of suitable diagnostic methods in order to gain synergic effect as a result of combination of conventional methods of technical diagnostics utilized for certain group of production machines, and monitoring of technological and non-technological parameters related to production processes. Each method of technical diagnostics and monitoring of production process is characteristic not only by measured parameters but also physical phenomenon used for its measurement and also by characteristics and volume of the measured data and signals that have to be stored for evaluation. The purpose of integration of muticriterial diagnostics is optimization of diagnostics of production processes and setting of diagnostic processes in production in way that allows maximal positive effect on stability of production processes thru maintenance planning $[8,11]$.

Keeping availability of diagnostic tools in mind, we expect the usage of multiple available methods such as measurement of vibration in various places of production device, in multiple axes during its operation and taking sources of mechanical wave motion and its spreading into consideration. Vibrodiagnostics as part of multicriterial diagnostics offers significant amount of information about the state of monitored object, such as changes of state, its character and localization. Same time, vibrodiagnostics allows certain amount of automatizability, what simplifies 
its implementation to long-term and short-term monitoring and also into multicriterial diagnostics. Particularly interesting results can be gained by measurement and analysis of characteristic vibrations of spindle of machine tools. In terms of the methods of signal processing and the volume of data gained during measurement, vibrodiagnostics is similar to noise analysis, which differ mainly by used sensors, where microphones are used instead of vibration sensors. The recorded sound is processed through various filters in order to extract usable signal which can be scanned for distinctive patterns afterwards. Such patterns allow to determine corresponding events. Vibrodiagnostics provides a wide range of information about the condition of machinery, but it is unable to reflect the condition completely. Vibrodiagnostics is specific by significant amount of measured data what can complicate the processing.

Another methods with high potential in multicriterial diagnostics, we determined, the thermography and measurement of temperature of constriction nodes of the monitored objects. The increased thermal emissions and related increase of temperature of the component is usually directly related to increased friction, load or electrical resistivity as result of mechanical or electrical failure of monitored device. The first analysis allows to identify the nodes where change of temperature can occur as a result of various events and localize positions where temperature sensors can be placed in order to perform long-term monitoring and diagnostics. Measurement of temperature of individual nodes can be relatively easy to implement into common production devices with no effect to its original purpose and functionality. The measured data can be easily analysed directly in real time or in the context of activities carried out by monitored devices with further increase of the reliability and sensitivity to changes that may occur at various levels and type of loads on the monitored device.

The engineering industry uses dimensional geometric accuracy as one of the most important parts of the quality of production. Accuracy of products, not only machined parts, is directly linked to precision of production device and therefore, precision of positioning and its analysis should not be neglected in the field of multicriterial diagnostics, even though it can be hardly implemented directly into autonomous systems. The alternative to fully automated system for monitoring is the implementation of measurement of positioning precision at regular intervals or at occurrence of event of change of the monitored parameter or parameters. This requires specification of certain limits for monitored parameters that requires repeated measurement of positioning precision. The implementation of precision measurement of production devices anticipates the usage of laser interferometer and device for the analysis of precision of positioning during circular interpolation. The described measurements give information about complex technical state of the observed production device as technical system and its subsystems that are involved in positioning.

Precision of machinery is closely related to wear. The wear is usually not uniform along all the effective workspace of a machine tool and therefore, it is possible to say that precision is the function of position. Wear is directly linked to usage rate, so maximal wear is usually localized at most exposed places and vice-versa, the fewer used parts of workspace should keep maximal precision longer. The mentioned facts indicate that the monitoring of the working space of production device can be used as a base for prediction of future wear of movement mechanisms based on usage rate of different parts of workspace. Besides of position there are other factors that have a considerable impact on wear rate of machine such as forces produced during normal operation of device and therefore, it is important to monitor other parameters such as spindle speed and federate in individual axes. Next parameter suitable for long-term monitoring which can easily reveal some aspects of machine state is the amount of electric energy consumed. If the amount of electricity necessary to perform specific action rises, it usually indicates that energy is consumed to overcome previously absent resistance or obstacle. The data received by described monitoring will be included into the basis of the analyzed data. Important part of the following analysis will be revealing correlations between parameters obtained by multicriterial analysis and optimization of data harvesting processes accordingly.

Base methodology to deal with the second objective is the implementation of a method to preserve data obtained in the process of multicriteria diagnostics, implementation of relevant tools and consequently processing and evaluating the obtained data through analytical tools. The results will be presented to final customer through suitable informative views.

Given the scope and nature of the data obtained during the implementation of multi-criteria diag- 
nostics while respecting the need for rapid storage and processing measured data we propose to use database application approach. If deployed into the production environment on the corresponding sufficient hardware, it gives us high-performance set of tools that will allow us to perform all the required tasks associated with storage of the measured (raw) data without restrictions due to different structuring of the stored data and it will allow us to back up all the required data, and pre-processing of data on the data server, without sacrificing the performance on computers that will implement the analytical processes, enabling us to flexibly create and modify the rights to stored data, and allow us to be very responsive when providing a response to a query processed by set of data analysis tools. An important part of the database system to allow processing of high volume data is a tool for data optimization and data processing tools to maintain data integrity.

The complexity of multi-criteria diagnostics is proportionally depending on the number of data collected in the production process. They are the parameters describing the status of individual elements that are part of every production process (machinery, tools, workpiece, etc.), but also elements indirectly affecting the production process (alignment of machines, cutting tool abrasion, state of coolants, etc.), and elements that externally influence the process of manufacture (ambient temperature, ambient humidity, the state of power network, etc.). As the number of monitored elements is growing, the number of endpoints increases proportionally and this causes proportional increase in data acquisition.
The data obtained during the multi-criteria diagnostics in the manufacturing process are usually very large in volume. Likewise, we can say that when monitored data is properly defined the data obtained during the multi-criteria diagnostics give us very large amount of usable informational. Our role is to provide understanding of the data obtained in the contexts of the monitored production process, detect cross-correlation between the parameters of the production process and enable to the user of our system to optimality access the presented information, by correctly defining the set of interpretation rules. To achieve these objectives, we need to have powerful analytical tools that allow us to process all necessary steps with the available data obtained by the multi-criteria diagnostics.

\section{APPLICATION OF ANALYTICAL INTELLIGENCE METHODS}

For this reason, we propose to use in obtaining the necessary information tools designed to analyse large volumes of data, this tools are sometimes known as Analytical Intelligence tools. They can be defined as tools that arose from a set of concepts and methodologies for improving decision-making process based on the use of metrics and the use of systems-based metrics. Analytical Intelligence Tools (AIT) is an important factor affecting the quality of analytical informatics processes in the competitiveness of enterprises. The basic principles of ATI include data transformation from databases and production systems

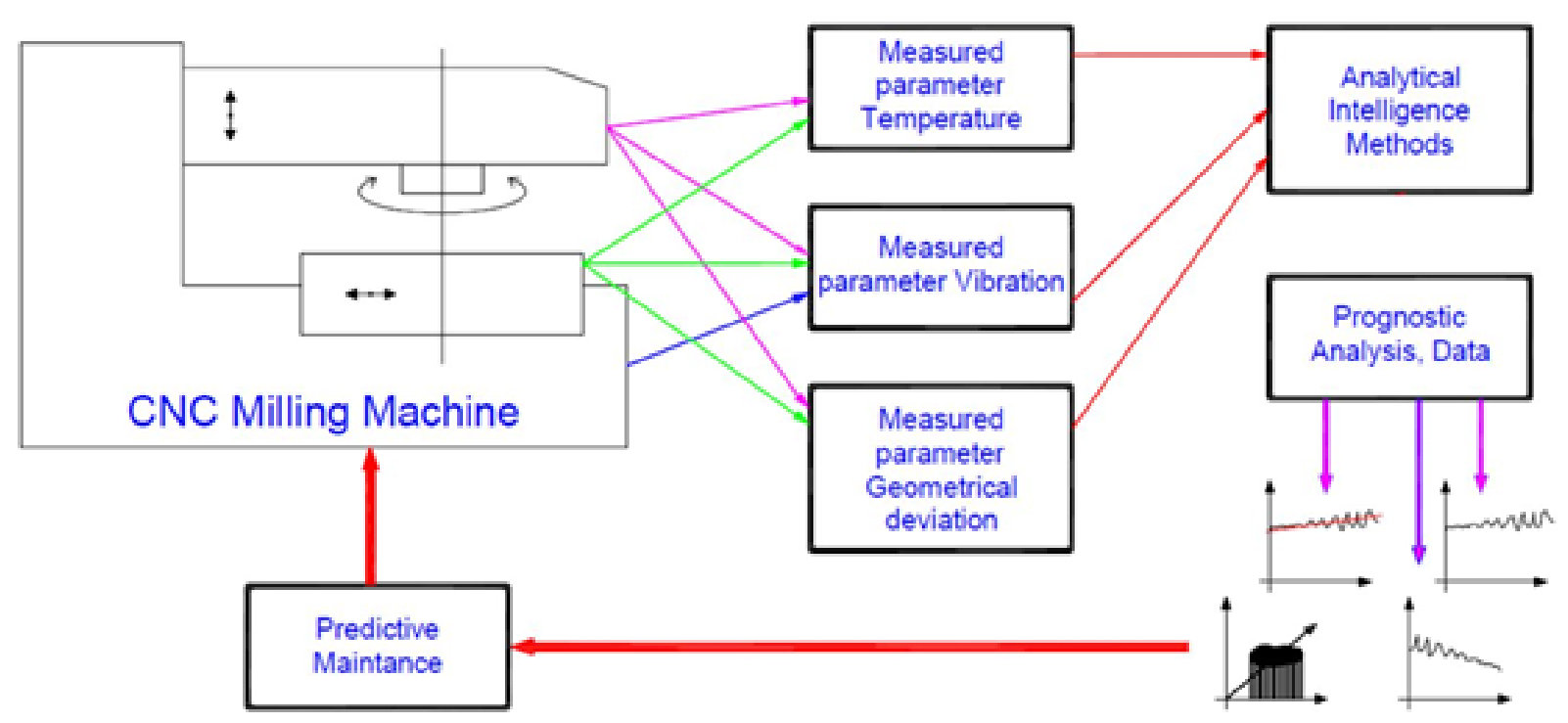

Fig. 3. Scheme of utilisation of analytical intelligence tools for multicriterions diagnostics of CNC machine tool 
to the analytical data. ATI solutions are based on multidimensionality in stored and manipulated data. ATI methods are based on Business Intelligence methods utilised in economical domain where also a lot of inputs.

Figure 3 introduces the principle of utilisation of analytical intelligence as methods for multicriterions diagnostics. The measured data such as temperature, vibrations and geometrical properties from various units of $\mathrm{CNC}$ machine tools will be assessed together and give complex perception about individual influences of measured data on technical condition of machine (fig.3). The output of analytical intelligence are prognosis of precision of machine tool in time. This output is very good and relevant input data for predictive maintenance. The manager is able to predict technical condition of machines and the maintenance planning is based on real machine condition.

Analytical intelligence are based on the following methods:

- on-line evaluation of input data,

- making prognosis and analysis report,

- data mining methods,

- online analytical processing (OLAP) and making OLAP cube,

- multi-dimensional array of data,

- making hypercube,

- obtaining a coherence among lot of measured data and output parameters,

- combination of processing of historical and actual data from production.

The focus of our research in the transformation of data collected from multi-criteria diagnostics to information presented to the end user will be primarily done by using tools for concurrent processing multidimensional data of AIT tools. From OLAP tools we will focus on defining data structures that are also known as OLAP cube, which allow us to carry out necessary analysis on the data gathered my multi-criteria diagnostics, for effective analytical planning.

Planned analytical process will be carried out through data mining. Data mining will be realized through statistical methods and artificial intelligence that will allow us to find correlations and other hidden or unknown dependencies between data acquired by multi-criteria diagnostics. Subsequently, the results of analysis will be processed through predictor models or segmentation models and then presented in a form of the results to the end user.

On the basis of the described methodology to deal with defined roles and selection of tools that we plan to apply in our research we are confident to say that they are designed competently and with emphasis on the ability to meet the declared objectives in full.

\section{REFERENCES}

1. Witten I. Data mining. Morgan Kaufman Publishers, 2011.

2. Cheng K. Machining dynamics. Springer, 2009.

3. Shirinzadeh B. and Teoh P.L. Laser interferometrybased guidance methodology for high precision positioning of mechanisms and robots. Robotics and Computer-Integrated Manufacturing, 26, 2010, 74-82.

4. Lovicz R. and Dalley R. Wear particle analysis - a predictive maintenance tool. The Predictive Maintenance Technology Conference, 2006.

5. Castroa H.F.F. and Burdekinb M. Calibration system based on a laser interferometer for kinematic accuracy assessment on machine tools. Inter. Journal of Machine Tools and Manufacture, 46, 2006, 89-97.

6. Jozwik J. and Kuric I. Bezkontaktowe systemy diagnostyczne obrabiarek sterowanych numerycznie CNC (in Polish). 14th Intern. Conference Automation in Production Planning and Manufacturing, Zilina, 2013.

7. Majda P. The influence of geometric errors compensation of a CNC machine tool on the accuracy of movement with circular interpolation. Advances in Manufacturing Science and Technology, 36(2), 2012, 59-67.

8. Stancekova D., Semcer J., Rudawska A. and Cep R. Identification of drilling of biocompatible materials based on titanium. Manufacturing Technology, 15(4), 2015, 699-704.

9. Nakazawa H. and Ito K. Measurement system of contouring accuracy on NC machine tools. Bull. Japan Soc. Prec. Eng., 12(4), 1978, 189.

10. Saga M. et al. Advanced methods in computational and experimental mechanics. Pearson Education Limited, London, United Kingdom, 2013, 57-112.

11. Rudawska A., Cubonova N., Pomaranska K., Stancekova D. and Gola A. Technical and organizational improvements of packaging production process. Advances In Science And TechnologyResearch Journal, 10(30), 2016, 182-192.

12. Medik S. Motion errors. Introduction to Precision Machine Design and Error Assessment. Edited by Samir Mekid, CRC Press, 2008, 9-73.

13. Sapietova A., Saga M. and Novak P. Multi-software platform for solving of multibody systems synthesis. Communication - Scientific Letters of the University of Zilina, 14(3), 2012, 43-48. 